\title{
New perspectives on the roles of circular RNAs in osteoarthritis development and progression (Review)
}

\author{
GUODING CAO ${ }^{1,2}$, YUQI PEI ${ }^{3}$, PENG $\mathrm{LI}^{2,3}$, PENG LIU $^{2}$, YAPENG DENG $^{2,4}$, \\ $\mathrm{YU} \mathrm{GAO}^{2,5}$, JUN LIU ${ }^{6}$ and XUSHENG $\mathrm{LI}^{2}$
}

\begin{abstract}
${ }^{1}$ Department of Orthopedics, Affiliated Hospital of Gansu Medical College, Pingliang, Gansu 744000;
${ }^{2}$ Department of Orthopedics, The 940th Hospital of the Joint Logistics Support Force of the Chinese People's Liberation Army, Lanzhou, Gansu 730050; ${ }^{3}$ Gansu University of Traditional Chinese Medicine, Lanzhou, Gansu 730000; ${ }^{4}$ School of Medicine, Lanzhou University, Lanzhou, Gansu 730000; ${ }^{5}$ School of Medicine, Ningxia Medical University, Yinchuan, Ningxia 750000;

${ }^{6}$ Department of Orthopedics, The Second Affiliated Hospital of Xi'an Jiaotong University, Xi'an, Shanxi 710061, P.R. China
\end{abstract}

Received May 1, 2021; Accepted August 24, 2021

DOI: $10.3892 /$ etm.2021.10906

\begin{abstract}
Osteoarthritis (OA) is a common disease of the elderly, posing a major personal and socioeconomic burden. $\mathrm{OA}$ is characterized by painful degeneration of articular cartilage, and its prevention, diagnosis and treatment remain problematic. Circular RNAs (circRNAs) constitute a large family of non-coding RNAs that are widely distributed, stable, conserved and tissue-specific. circRNAs have been found to be closely associated with OA development and progression, and they may serve as targets for disease prevention and treatment. The aim of the present article was to review the roles of circRNAs in OA and discuss possible treatment strategies.
\end{abstract}

\section{Contents}

1. Introduction

2. circRNA characteristics

3. circRNA functions

4. Roles of circRNAs in OA

5. circRNAs and OA treatment

6. Conclusion and perspectives

Correspondence to: Professor Jun Liu, Department of Orthopedics, The Second Affiliated Hospital of Xi'an Jiaotong University, 157 Xiwu Road, Xincheng, Xi'an, Shanxi 710061, P.R. China

E-mail: tutuhehtt@126.com

Professor Xusheng Li, Department of Orthopedics, The 940th Hospital of the Joint Logistics Support Force of the Chinese People's Liberation Army, 333 Binhe South Road, Qilihe, Lanzhou, Gansu 730050, P.R. China

E-mail: lixush@sina.com

Key words: osteoarthritis, chondrocyte, extracellular matrix, circular RNA

\section{Introduction}

Osteoarthritis (OA) is an atypical degenerative disease of articular cartilage that can lead to progressive pain, joint dysfunction and general disability (1), particularly in the elderly population, and the disease incidence is increasing worldwide along with population aging. OA renders exercise difficult, diminishes the quality of life of those affected (2) and imposes a major socioeconomic burden. The pathogenesis of OA is affected by age, sex, obesity, abnormal osteoclast and chondrocyte activities, and a dysfunctional extracellular matrix (ECM) (3). However, OA prevention, diagnosis and treatment are inadequate. Treatment mainly focuses on pain relief using conventional medications (4), intra-articular steroid injections $(5,6)$ and surgery, but treatment efficacy and patient prognosis remain unsatisfactory. Therefore, new approaches are urgently required. Circular RNAs (circRNAs) serve important roles in $\mathrm{OA}$, and they may provide new research directions in terms of prevention, diagnosis and treatment. The focus of the present review was on the characteristics of circRNAs and their involvement in OA, as circRNA targeting may prove useful as a treatment approach and may also help identify new areas for research.

\section{2. circRNA characteristics}

The structure and functions of circRNAs are becoming increasingly understood. circRNAs are abundant and ubiquitous in nature, and $>10,000$ different circRNAs have been identified in human cells to date (4). The expression levels of some circRNAs are higher compared with those of their linear isotypes $(7,8)$. circRNAs are very stable, with the half-lives of most exceeding $48 \mathrm{~h}$ (7), which is longer compared with the half-lives of mammalian mRNAs. circRNAs are covalently closed loops, lacking 5' caps and 3' tails, which makes them resistant to the shearing action of RNA exonuclease and ribonuclease R (9). Compared to linear RNAs, circRNAs are more highly conserved, more tissue-specific and more confined to particular subcellular compartments $(10,11)$. Most exon circRNAs are found in the cytoplasm (12), whereas most intron 
and intron-exon circRNAs are found in the nucleus (12-14). circRNAs may serve as therapeutic targets and they may be produced in large amounts if required.

\section{3. circRNA functions}

The old view held that the production of circRNAs was merely the result of accidental splicing and had no actual biological function. However, later studies have shown that circRNAs are not products of accidental splicing. Indeed; circRNAs have some specific formation process models, including spliceosomal mediated reverse splicing and linking; processing of exons and flanking introns; the regulatory of RNA binding proteins. circRNAs may act as microRNA (miRNA/miR) sponges (15), interact with transcription regulators and RNA-binding proteins, and they can be translated into proteins. miRNAs are non-coding RNAs $\sim 20$ nucleotides in length (16). The binding of miRNAs to the 3'-untranslated regions of mRNAs inhibits translation and, thus, gene expression (17). Certain circRNAs, such as ciRS-7/CDR1as and SRY RNA, have been shown to serve as miRNA sponges (18). The mouse brain ciRS-7/CDR 1 circRNA features $>70$ miRNA-binding sites (19), whereas SRY RNA has 16 binding sites (20). Another miRNA sponge, circHIPK3, has 18 binding sites for miR-124, thus inhibiting the antiproliferative effect of miR-124 in malignant tumors and protecting chondrocytes against $\mathrm{OA}$; in addition, circHIPK 3 was shown to bind to miR-30a and miR-558 (21-23). The sponging effects of the circRNA_33186/miR-127-5p combination strongly enhance pathogenicity- circRNA_33186 directly binds and inhibits miR-127-5p, thereby increasing the expression of MMP-13 and contributing to the pathogenesis of OA (24). circRNAs also transcriptionally regulate their parent genes (24). The intron circRNA ci-ankrd52 and the exon-intron circRNAs circ-EIF3J and circ-PAIP2 interact with the RNA-encoding Pol II to regulate gene expression $(13,14)$. circRNAs interact with RNA-binding proteins (RBPs), forming RNA-protein complexes that regulate post-transcriptional RNA events (25). The circRNA/RBP effects are very similar to those of miRNA sponges; circRNA binding to RBPs reduces RBP/RNA interactions. For example, circ-like non-coding RNAs [exon-intron circRNAs (EIciRNAs)] specifically interact with RNAs encoding antibodies targeting the U1 small ribonucleoprotein (snRNP), forming EIciRNA-U1-snRNP complexes that recruit RNA Pol II, thus promoting cis-mediated activation (enhanced transcription) of the gene encoding that enzyme (25). RBPs negatively affect RNA cyclization. For example, some adenosine deaminases acting on RNA (ADARs) are in fact RBPs featuring RNA-editing functions that disrupt RNA stem structures and, thus, restrict circRNA formation. ADAR knockdown promotes circRNA expression (26). In addition, circRNAs may be translated into proteins in a manner that differs from the translation of linear mRNAs. circRNAs lack 5 ' caps; thus, translation is cap-independent, exploiting open reading frames featuring internal ribosomal entry sites (27) or 5'-untranslated regions that contain N6-methyladenosine (28).

The aforementioned findings may provide some context for circRNA research and applications in the OA context, but the roles played by circRNAs in OA require further clarification.

\section{Roles of circRNAs in OA}

circRNAs interact with chondrocytes (Fig. 1). Chondrocyte apoptosis (triggered through various pathways) is a crucial feature of OA (29). circRNAs have been reported to regulate chondrocyte apoptosis and proliferation. For example, circADAMTS6 overexpression was shown to inhibit human chondrocyte apoptosis, whereas miR-431-5p overexpression exerted the opposite effect. circADAMTS6 sponges miR-431-5p to create a circADAMTS6/miR-431-5p axis that regulates IL-1 $\beta$-induced chondrocyte apoptosis (30). circHIPK3 is negatively correlated with miR-124. Promoting miR-124, thus suppressing SOX8 expression, OA chondrocyte apoptosis can be promoted by low-level circHIPK3 expression (31). Therefore, circHPIK3 may exert protective effects on chondrocytes in an osteoarthritic environment. circANKRD36 knockout was shown to promote IL-1 $\beta$-induced apoptosis and chondrocyte inflammation (32). The zinc-finger-containing transcription factor Casz1 prevents chondrocyte apoptosis and IL-1 $\beta$-mediated inflammation; the sponging interaction between circANKRD36 and miR-599 upregulated Casz1 expression, in turn preventing chondrocyte apoptosis and inflammation in OA (32). circ_0092516 regulates proliferation and apoptosis of OA chondrocytes by controlling the miR-337-3p/PTEN axis; circ_0092516 binds to miR-337-3p and, thus, modulates the effects of the miR-337-3p/PTEN axis, promoting chondrocyte proliferation, inhibiting chondrocyte apoptosis, and delaying OA progression (33).

However, circRNAs can also damage chondrocytes. For example, circ_0114876 was shown to regulate TNF receptor-associated factor 2 (TRAF2) expression in IL-1 $\beta$-induced CHON-001 cells by sponging miR-671, thereby controlling the damage of chondrocytes in OA progression and the inflammatory response, and to promote chondrocyte injury by targeting the miR-671/TRAF2 axis that is activated following IL-1ß-induced injury (34). Hsa_circ_0005567 can activate autophagy by regulating the miR-495/Atg14 axis, thus inhibiting IL-1 $\beta$-induced chondrocyte apoptosis (35). Chondrocyte viability is closely associated with the development of OA. The findings presented above suggested that circRNA targeting may enhance chondrocyte proliferation or reduce apoptosis, thereby improving OA.

\section{circRNAs regulate the chondrocyte ECM}

circRNAs regulate metabolic homeostasis in chondrocyte $E C M$. OA is typically accompanied by progressive cartilage loss within the entire joint structure $(36,37)$. The most important components of the ECM are type II collagen (which imparts tensile strength) and aggrecan (which renders cartilage compression-resistant) $(3,38)$. Anabolism and catabolism are in dynamic equilibrium in healthy ECM (38), but not under disease conditions. Early in OA progression, chondrocytes repair damaged cartilage, during which time cartilage matrix synthesis and chondrocyte proliferation are vigorous (39). Some circRNAs promote the synthesis of cartilage matrix proteins. For example, circPDE4D serves as a sponge for miR-103a-3p; as miR-103a-3p directly targets fibroblast growth factor (FGF)18 expression, circPDE4D binding to miR-103a-3p regulates FGF18 synthesis and, thus, protects against OA by maintaining the ECM (40). circCDK14 serves 


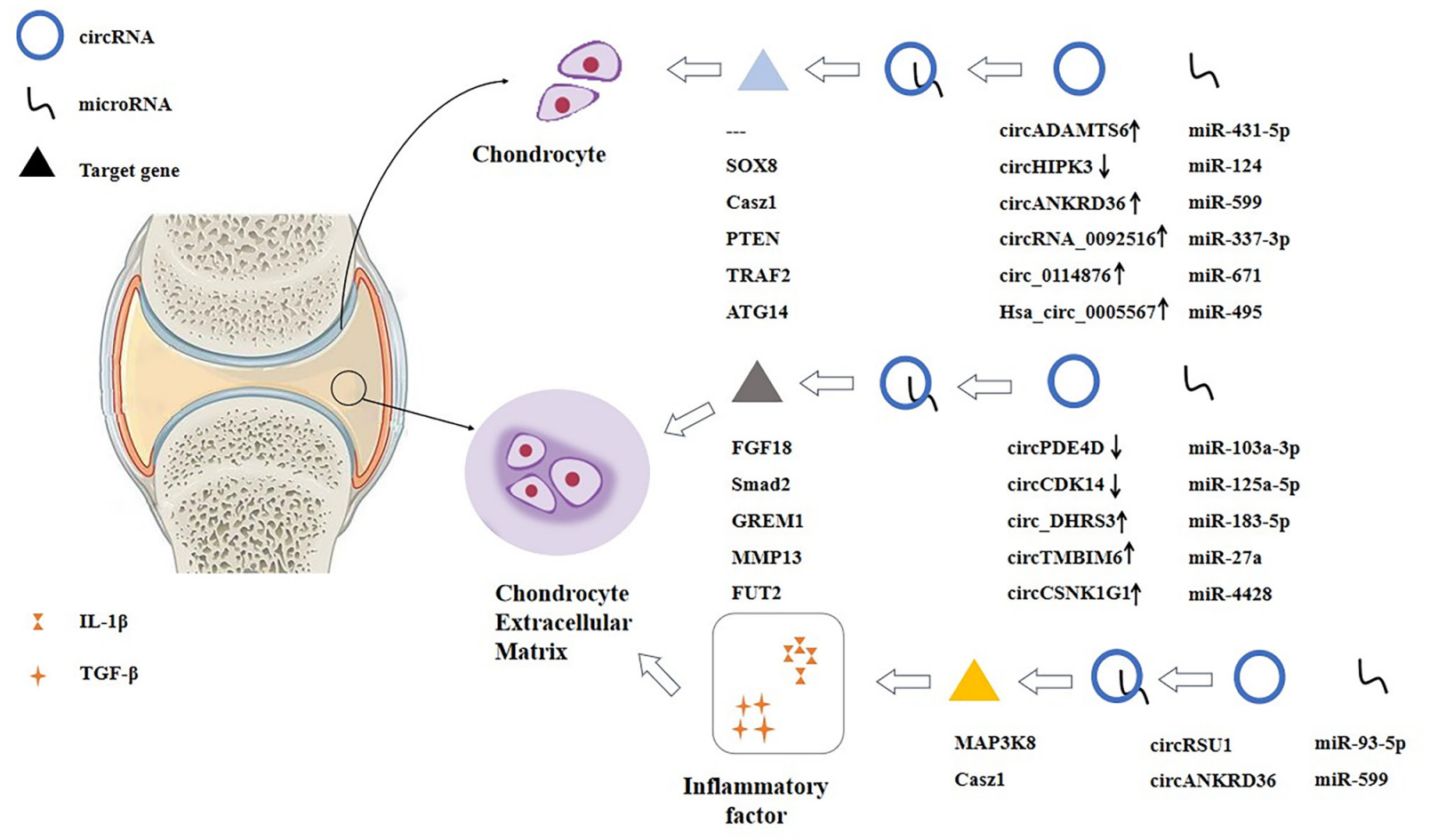

Figure 1. Schematic illustration of the role of circRNAs in osteoarthritis. circRNAs interact with chondrocytes, regulate metabolic homeostasis and the inflammatory response of the cartilage ECM, and greatly influence osteoarthritis development and progression. CircRNA, circular RNA; FGF, fibroblast growth factor; FUT2, fucosyltransferase 2; GREM, gremlin; miR, microRNA; TRAF2, TNF receptor-associated factor 2

as an miR-125a-5p sponge, downregulating Smad2 expression and inhibiting TGF- $\beta$ signaling, thereby protecting the chondrocyte ECM (41).

However, certain circRNAs may promote ECM degradation. For example circ_DHRS3 increases Gremlin 1 expression by competitively binding miR-183-5p to regulate IL-1 $\beta$-regulated ECM degradation (42). It was recently demonstrated that circCSNK1G1 upregulation is also associated with ECM degradation. circCSNK1G1 and fucosyltransferase 2 (FUT2) induce ECM degradation and chondrocyte apoptosis; miR-4428 targets FUT2 mRNA to inhibit its expression, reversing the negative effects of circCSNK1G1 and FUT2 (43). CircCSNK1G1 was upregulated in OA affected cartilage, which can induce chondrocyte apoptosis and EMC degradation through sponging mir-4428 targets. miR-4428 conferred a protective effect on OA by inhibiting FUT2 expression. circTMBIM6 promotes cartilage ECM degradation induced by OA via the circTMBIM6/miR-27a/MMP13 axis (44). Thus, circRNAs either promote or inhibit OA occurrence and development; targeting of the aforementioned circRNAs may enhance ECM homeostasis, thereby alleviating OA.

circRNAs regulate ECM inflammation. As OA progresses, the levels of inflammatory mediators, including cytokines, chemokines, prostaglandins and leukotrienes, increase in affected joints and the synovial fluid (38). A number of studies have described the involvement of circRNAs in these processes. Yang et al (45) found that circRSU1 was induced by IL- $1 \beta$ and $\mathrm{H}_{2} \mathrm{O}_{2}$, and then modulated oxidative stress-induced inflammation and ECM dysfunction by regulating the MEK/Erk1/Erk2 and NF- $\mathrm{KB}$ signaling cascades. The circRSU1/miR-93-5p/MAP3K8 axis increased the oxidative stress involved in OA progression; thus, this axis may serve as a useful therapeutic target (45). circANKRD36 levels are decreased in OA tissues, and circANKRD36 knockout promoted apoptosis and inflammation of chondrocytes that were under IL-1 $\beta$-induced stress. miR-599 directly targets Casz1 to prevent chondrocyte apoptosis and inflammation caused by IL-1 $\beta$. circANKRD36 protects against OA by upregulating Caszl expression through sponging miR-599 (32).

circRNAs are also involved in innate immunity; the circRNA profiles of M1 and M2 macrophages were found to be different (46), suggesting potential roles for circRNAs in macrophage differentiation and polarization. circRasGEF1B has been found to regulate macrophage activation. OA progression reflects activation of the innate immune response $(47,48)$, and various cells of the innate immune system (including monocytes/macrophages and mast cells) become enriched (49). circRNAs may influence these processes, thus modulating the OA course (50); however, this remains conjectural. Inflammation triggers intermittent and progressive joint pain, and the focus of OA treatment is mainly pain relief, which is often ineffective (Table I).

\section{5. circRNAs and OA treatment}

As OA progresses, various circRNAs become up- or downregulated and they may serve as prognostic markers or therapeutic targets. The levels of hsa_circ_101178 in serum and synovial fluid were found to be significantly higher in patients with OA compared with those in control patients; the serum level was positively correlated with OA severity, so that level can be used to predict OA development (51). As explained above, targeting 
Table I. Roles of circRNAs in osteoarthritis.

\begin{tabular}{|c|c|c|c|}
\hline Relevant context & circRNA & Sponged miRNA & Function \\
\hline \multirow[t]{5}{*}{$\begin{array}{l}\text { Associated with } \\
\text { chondrocytes }\end{array}$} & circADAMTS6 (upregulated) & $\operatorname{miR}-431-5 p$ & $\begin{array}{l}\text { circADAMTS6/miR-431-5p axis inhibits } \\
\text { apoptosis of human chondrocytes }\end{array}$ \\
\hline & circHIPK3 (downgrade) & miR-124 & Protective effect on chondrocytes in OA \\
\hline & circ_0092516 (upregulated) & $\operatorname{miR}-337-3 p$ & $\begin{array}{l}\text { miR-337-3p/PTEN axis promotes } \\
\text { chondrocyte proliferation }\end{array}$ \\
\hline & circ_0114876 (upregulated) & miR-671 & $\begin{array}{l}\text { miR-671/TRAF2 axis promotes } \\
\text { chondrocyte injury }\end{array}$ \\
\hline & circ_0005567 (upregulated) & miR-495 & $\begin{array}{l}\text { miR-495/Atg } 14 \text { axis activates autophagy } \\
\text { and inhibits chondrocyte apoptosis }\end{array}$ \\
\hline \multirow{5}{*}{$\begin{array}{l}\text { Metabolic homeostasis } \\
\text { of the ECM }\end{array}$} & circPDE4D (downregulated) & miR-103a-3p & Modulates protective role of FGF18 \\
\hline & circCDK14 (downregulated) & $\operatorname{miR}-125 a-5 p$ & $\begin{array}{l}\text { Downregulates the expression of SMAD2 } \\
\text { and protects the chondrocyte ECM }\end{array}$ \\
\hline & circ_DHRS3 (upregulated) & $\operatorname{miR}-183-5 p$ & $\begin{array}{l}\text { Regulates the degradation of ECM positively } \\
\text { regulating the expression of gremlin } 1\end{array}$ \\
\hline & circTMBIM6 (upregulated) & $\operatorname{miR}-27 \mathrm{a}$ & $\begin{array}{l}\text { circTMBIM6/miR-27a/MMP13 axis } \\
\text { degrades chondrocyte ECM }\end{array}$ \\
\hline & circCSNK1G1 (upregulated) & $\operatorname{miR}-4428$ & $\begin{array}{l}\text { circCSNK1G1 and FUT2 can induce ECM } \\
\text { degradation and chondrocyte apoptosis, } \\
\text { while miR- } 4428 \text { reverses the negative effects } \\
\text { of circCSNK1G1 and FUT2 }\end{array}$ \\
\hline \multirow[t]{3}{*}{ Inflammation of the ECM } & circRSU1 (upregulated) & miR-93-5p & $\begin{array}{l}\text { circRSU1/miR-93-5p/MAP3K8 axis } \\
\text { regulates oxidative stress in progressive OA }\end{array}$ \\
\hline & circANKRD36 (upregulated) & miR-599 & $\begin{array}{l}\text { circANKRD36 upregulates the expression of } \\
\text { Casz1 against miR-599 }\end{array}$ \\
\hline & circRasGEF1B (upregulated) & $\operatorname{miR}-21-3 p$ & $\begin{array}{l}\text { circRasGEF1B can counteract the effect of } \\
\text { miR-21-3p, enhancing the innate } \\
\text { antiviral response }\end{array}$ \\
\hline Associated with treatment & $\begin{array}{l}\text { circ_0083429 (upregulated) } \\
\text { circCDH13 (upregulated) }\end{array}$ & $\begin{array}{l}\operatorname{miR}-346 \\
\operatorname{miR}-296-3 p\end{array}$ & $\begin{array}{l}\text { miR-346/Smad3 axis alleviates OA } \\
\text { circcDH13/miR-296-3p/PTEN axis } \\
\text { promotes OA }\end{array}$ \\
\hline
\end{tabular}

circRNA, circular RNA; ECM, extracellular matrix; FGF, fibroblast growth factor; FUT2, fucosyltransferase 2; miR, microRNA; OA, osteoarthritis; TRAF2, TNF receptor-associated factor 2.

of circ_DHRS3 and circPDE4D may alleviate OA. circ0083429 regulates Smad3 by sponging miRNA-346, thereby alleviating OA through the miR-346/Smad3 axis (52). circCDH13 promotes OA by sponging miR-296-3p, contributing to OA pathogenesis via the circCDH13/miR-296-3p/PTEN axis (53). circRNAs may thus induce the differentiation of cells that target OA. Stem cells (SCs) from the bone marrow or adipose or other potential tissues, such as the umbilical cord, can differentiate into chondrocytes that repair tissue damage (54-56). During such differentiation, the expression levels of several circRNAs are altered. For example, circRNA-CDRlas can maintain human umbilical cord SC (husMSCs) differentiation and proliferation capacities. The knockdown of CDRlas resulted in the down-regulation of the expression of dry transcription factors (STFs), which impaired the osteogenic potential of hucMSCs. In addition, circRNA may maintain SC chondrogenic differentiation, thus promoting differentiation that aids bone and joint function (57).
circRNAs can also be loaded into vesicles that are then transported to specific bodily sites (58). Extracellular vesicles are important regulators of cartilage homeostasis and OA; circRNAs within such vesicles may thus exert useful effects. Exosomes are lipid membrane-type extracellular vesicles formed through endocytosis, integration and efflux, and they are found in numerous biological fluids, such as blood, urine, saliva and cerebrospinal fluid (59-61). Recently, exosomes have become favored drug carriers owing to their nanoscale dimensions, low immunogenicity, ability to penetrate biofilms and convenient storage (62). circRNAs can be loaded into exosomes (63), but few relevant studies have been published to date. Yang et al (64) reported that injecting engineered rabies virus glycoprotein-circSCMH1-extracellular vesicles rabies virus glycoprotein-circSCMH1-extracellular vesicles into the brain improved stroke outcomes in mice and monkeys. However, OA data are lacking, as access to OA 
tissue is difficult, ethical considerations may be problematic, the cost of such research is high and good outcomes are not assured (65).

\section{Conclusion and perspectives}

circRNAs serve key roles in OA development and progression; as such, circRNA targeting may prevent OA or stop disease progression. The present review discussed circRNAs in general and their possible roles in OA. It appears that circRNAs may be used to treat OA, but further extensive research remains to be performed. circRNAs are abundant, stable, highly conserved and tissue-specific. circRNAs sponge miRNAs $(18,20)$; however, other effects remain poorly understood. Currently, most studies on the role of circRNAs in OA focus on metabolic homeostasis, cell apoptosis and inflammation, whereas the effects of circRNAs on chondrocytes and stromal cells, the immune microenvironment and gene regulation require further study. Therefore, circRNAs may serve as OA biomarkers and therapeutic targets, but additional investigation is warranted. OA is a complex disease, and a single anti-circRNA treatment may not be efficacious. Targeting of several circRNAs may be essential when seeking to prevent disease development, arrest progression and improve treatment.

\section{Acknowledgements}

Not applicable.

\section{Funding}

The present study was supported by The Military Logistics Research Project (grant no. CWH17J009); The Military Medical Science and Technology Youth Development Program (grant no. 19QNP047); The Youth Science and Technology Foundation of Gansu Province (grant no. 1606RJYA300); The Health Industry Research Plan Project of Gansu Province (grant no. GSWSKY2018-21); The Natural Science Foundation of Gansu Province (grant no. 1606RJZA208); and The Lan'Zhou Talent Innovation and Entrepreneurship Project (grant no. 2019-RC-65), P.R. China.

\section{Availability of data and materials}

Not applicable.

\section{Authors' contributions}

GC contributed to the investigation and wrote the original draft of the manuscript. YP and PLi performed the relevant literaure research and revised the manuscript. PLiu, YD, YG and JL contributed to literature searching and processing. XL contributed to the conceptualization of the review. Data authentication is not applicable. All authors read and approved the final manuscript.

\section{Ethics approval and consent to participate}

Not applicable.

\section{Patient consent for publication}

Not applicable.

\section{Competing interests}

The authors declare that they have no competing interests.

\section{References}

1. Neogi T: The epidemiology and impact of pain in osteoarthritis. Osteoarthritis Cartilage 21: 1145-1153, 2013.

2. Barbour KE, Helmick CG, Boring $M$ and Brady TJ: Vital Signs: Prevalence of Doctor-Diagnosed Arthritis and Arthritis-Attributable Activity Limitation - United States, 2013-2015. MMWR Morb Mortal Wkly Rep 66: 246-253, 2017.

3. Glyn-Jones S, Palmer AJR, Agricola R, Price AJ, Vincent TL, Weinans H and Carr AJ: Osteoarthritis. Lancet 386: 376-387, 2015.

4. Taruc-Uy RL and Lynch SA: Diagnosis and treatment of osteoarthritis. Prim Care 40: 821-836, vii, 2013.

5. Hochberg MC, Altman RD, April KT, Benkhalti M, Guyatt G, McGowan J, Towheed T, Welch V, Wells G and Tugwell P; American College of Rheumatology: American College of Rheumatology 2012 recommendations for the use of nonpharmacologic and pharmacologic therapies in osteoarthritis of the hand, hip, and knee. Arthritis Care Res (Hoboken) 64: 465-474, 2012.

6. Rausch Osthoff AK, Niedermann K, Braun J, Adams J, Brodin N, Dagfinrud H, Duruoz T, Esbensen BA, Günther KP, Hurkmans E, et al: 2018 EULAR recommendations for physical activity in people with inflammatory arthritis and osteoarthritis. Ann Rheum Dis 77: 1251-1260, 2018.

7. Jeck WR, Sorrentino JA, Wang K, Slevin MK, Burd CE, Liu J, Marzluff WF and Sharpless NE: Circular RNAs are abundant, conserved, and associated with ALU repeats. RNA 19: 141-157, 2013.

8. Salzman J, Gawad C, Wang PL, Lacayo N and Brown PO: Circular RNAs are the predominant transcript isoform from hundreds of human genes in diverse cell types. PLoS One 7: e30733, 2012.

9. Suzuki $\mathrm{H}$ and Tsukahara T: A view of pre-mRNA splicing from RNase R resistant RNAs. Int J Mol Sci 15: 9331-9342, 2014.

10. Ivanov A, Memczak S, Wyler E, Torti F, Porath HT, Orejuela MR, Piechotta M, Levanon EY, Landthaler M, Dieterich C, et al: Analysis of intron sequences reveals hallmarks of circular RNA biogenesis in animals. Cell Rep 10: 170-177, 2015.

11. Du WW, Yang W, Chen Y, Wu ZK, Foster FS, Yang Z, Li X and Yang BB: Foxo3 circular RNA promotes cardiac senescence by modulating multiple factors associated with stress and senescence responses. Eur Heart J 38: 1402-1412, 2017.

12. Guo JU, Agarwal V, Guo H and Bartel DP: Expanded identification and characterization of mammalian circular RNAs. Genome Biol 15: 409, 2014.

13. Zhang Y, Zhang XO, Chen T, Xiang JF, Yin QF, Xing YH, Zhu S, Yang $L$ and Chen LL: Circular intronic long noncoding RNAs. Mol Cell 51: 792-806, 2013.

14. Li Z, Huang C, Bao C, Chen L, Lin M, Wang X, Zhong G, Yu B, $\mathrm{Hu}$ W, Dai L, et al: Exon-intron circular RNAs regulate transcription in the nucleus. Nat Struct Mol Biol 22: 256-264, 2015.

15. Thomas LF and Sætrom P: Circular RNAs are depleted of polymorphisms at microRNA binding sites. Bioinformatics 30: 2243-2246, 2014.

16. Krol J, Loedige I and Filipowicz W: The widespread regulation of microRNA biogenesis, function and decay. Nat Rev Genet 11: 597-610, 2010.

17. Bartel DP: MicroRNAs: Target recognition and regulatory functions. Cell 136: 215-233, 2009.

18. Memczak S, Jens M, Elefsinioti A, Torti F, Krueger J, Rybak A, Maier L, Mackowiak SD, Gregersen LH, Munschauer M, et al: Circular RNAs are a large class of animal RNAs with regulatory potency. Nature 495: 333-338, 2013.

19. Hansen TB, Wiklund ED, Bramsen JB, Villadsen SB, Statham AL, Clark SJ and Kjems J: miRNA-dependent gene silencing involving Ago2-mediated cleavage of a circular antisense RNA. EMBO J 30: 4414-4422, 2011. 
20. Hansen TB, Jensen TI, Clausen BH, Bramsen JB, Finsen B, Damgaard CK and Kjems J: Natural RNA circles function as efficient microRNA sponges. Nature 495: 384-388, 2013.

21. Zheng Q, Bao C, Guo W, Li S, Chen J, Chen B, Luo Y, Lyu D, Li Y, Shi G, et al: Circular RNA profiling reveals an abundant circHIPK 3 that regulates cell growth by sponging multiple miRNAs. Nat Commun 7: 11215, 2016.

22. Chen B, Yu J, Guo L, Byers MS, Wang Z, Chen X, Xu H and Nie Q: Circular RNA circHIPK3 promotes the proliferation and differentiation of chicken myoblast cells by sponging miR-30a-3p. Cells 8: E177, 2019.

23. Li Y, Zheng F, Xiao X, Xie F, Tao D, Huang C, Liu D, Wang M, Wang L, Zeng F, et al: CircHIPK3 sponges miR-558 to suppress heparanase expression in bladder cancer cells. EMBO Rep 18: 1646-1659, 2017.

24. Zhou ZB, Huang GX, Fu Q, Han B, Lu JJ, Chen AM and Zhu L: circRNA.33186 Contributes to the pathogenesis of osteoarthritis by sponging miR-127-5p. Mol Ther 27: 531-541, 2019.

25. Zhou Z, Du D, Chen A and Zhu L: Circular RNA expression profile of articular chondrocytes in an IL-1 $\beta$-induced mouse model of osteoarthritis. Gene 644: 20-26, 2018.

26. Shi L, Yan P, Liang Y, Sun Y, Shen J, Zhou S, Lin H, Liang X and Cai X: Circular RNA expression is suppressed by androgen receptor (AR)-regulated adenosine deaminase that acts on RNA (ADAR1) in human hepatocellular carcinoma. Cell Death Dis 8: e3171, 2017.

27. Legnini I, Di Timoteo G, Rossi F, Morlando M, Briganti F, Sthandier O, Fatica A, Santini T, Andronache A, Wade M, et al: Circ-ZNF609 is a circular RNA that can be translated and functions in myogenesis. Mol Cell 66: 22-37.e9, 2017.

28. Pamudurti NR, Bartok O, Jens M, Ashwal-Fluss R, Stottmeister C, Ruhe L, Hanan M, Wyler E, Perez-Hernandez D, Ramberger E, et al: Translation of CircRNAs. Mol Cell 66: 9-21.e7, 2017.

29. Kerr JF, Wyllie AH and Currie AR: Apoptosis: A basic biological phenomenon with wide-ranging implications in tissue kinetics. Br J Cancer 26: 239-257, 1972.

30. Fu Q, Li L, Wang B, Wu J, Li H, Han Y, Xiang D, Chen Y and Zhu J: CircADAMTS6/miR-431-5p axis regulate interleukin-1 $\beta$ induced chondrocyte apoptosis. J Gene Med 23: e3304, 2021.

31. Wu Q, Yuan ZH, Ma XB and Tang XH: Low expression of CircRNA HIPK3 promotes osteoarthritis chondrocyte apoptosis by serving as a sponge of miR-124 to regulate SOX8. Eur Rev Med Pharmacol Sci 24: 7937-7945, 2020.

32. Zhou JL, Deng S, Fang HS, Du XJ, Peng H and Hu QJ: Circular RNA circANKRD36 regulates Casz1 by targeting miR-599 to prevent osteoarthritis chondrocyte apoptosis and inflammation. J Cell Mol Med 25: 120-131, 2021.

33. Huang Z, Ma W, Xiao J, Dai X and Ling W: CircRNA_0092516 regulates chondrocyte proliferation and apoptosis in osteoarthritis through the miR-337-3p/PTEN axis. J Biochem 169: 467-475, 2021

34. Wang Q, Luo S, Yang J, Li J, Huan S, She G and Zha Z: Circ_0114876 promoted IL-1 $\beta$-induced chondrocyte injury by targeting miR-671/TRAF2 axis. Biotechnol Lett 43: 791-802, 2021.

35. Zhang J, Cheng F, Rong G, Tang Z and Gui B: Hsa_circ_0005567 activates autophagy and suppresses IL- $1 \beta$-Induced shondrocyte apoptosis by regulating miR-495. Front Mol Biosci 7: 216, 2020

36. Loeser RF, Goldring SR, Scanzello CR and Goldring MB: Osteoarthritis: A disease of the joint as an organ. Arthritis Rheum 64: 1697-1707, 2012.

37. Loeser RF: Molecular mechanisms of cartilage destruction in osteoarthritis. J Musculoskelet Neuronal Interact 8: 303-306, 2008.

38. Goldring MB: The role of the chondrocyte in osteoarthritis. Arthritis Rheum 43: 1916-1926, 2000.

39. Di Liu D, Liang YH, Yang YT, He M, Cai ZJ, Xiao WF and Li YS: Circular RNA in osteoarthritis: an updated insight into the pathophysiology and therapeutics. Am J Transl Res 13: $11-23,2021$.

40. Wu Y, Hong Z, Xu W, Chen J, Wang Q, Chen J, Ni W, Mei Z, Xie Z, Ma Y, et al: Circular RNA circPDE4D protects against osteoarthritis by binding to miR-103a-3p and regulating FGF18. Mol Ther 29: 308-323, 2021.

41. Shen P, Yang Y, Liu G, Chen W, Chen J, Wang Q, Gao H, Fan S, Shen $\mathrm{S}$ and Zhao X: CircCDK14 protects against osteoarthritis by sponging miR-125a-5p and promoting the expression of Smad2. Theranostics 10: 9113-9131, 2020.
42. Jiang R, Gao H, Cong F, Zhang W, Song $\mathrm{T}$ and $\mathrm{Yu} \mathrm{Z}$ Circ_DHRS3 positively regulates GREM1 expression by competitively targeting miR-183-5p to modulate IL-1 $\beta$-administered chondrocyte proliferation, apoptosis and ECM degradation. Int Immunopharmacol 91: 107293, 2021.

43. Xiao J, Wang R, Zhou W, Cai X and Ye Z: Circular RNA CSNK1G1 promotes the progression of osteoarthritis by targeting the miR 4428/FUT2 axis. Int J Mol Med 47: 232-242, 2021.

44. Bai ZM, Kang MM, Zhou XF and Wang D: CircTMBIM6 promotes osteoarthritis-induced chondrocyte extracellular matrix degradation via miR-27a/MMP13 axis. Eur Rev Med Pharmacol Sci 24: 7927-7936, 2020

45. Yang Y, Shen P, Yao T, Ma J, Chen Z, Zhu J, Gong Z, Shen $S$ and Fang $X$ : Novel role of circRSU1 in the progression of osteoarthritis by adjusting oxidative stress. Theranostics 11: 1877-1900, 2021.

46. Zhang Y, Zhang Y, Li X, Zhang M and Lv K: Microarray analysis of circular RNA expression patterns in polarized macrophages. Int J Mol Med 39: 373-379, 2017

47. Liu CX, Li X, Nan F, Jiang S, Gao X, Guo SK, Xue W, Cui Y, Dong K, Ding H, et al: Structure and degradation of circular RNAs regulate PKR activation in innate immunity. Cell 177: 865-880.e21, 2019.

48. Kalaitzoglou E, Griffin TM and Humphrey MB: Innate immune responses and osteoarthritis. Curr Rheumatol Rep 19: 45, 2017.

49. Klein-Wieringa IR, de Lange-Brokaar BJ, Yusuf E, Andersen SN, Kwekkeboom JC, Kroon HM, van Osch GJ, Zuurmond AM, Stojanovic-Susulic V, Nelissen RG, et al: Inflammatory cells in patients with endstage knee osteoarthritis: A comparison between the synovium and the infrapatellar fat pad. J Rheumatol 43: 771-778, 2016.

50. Zhang W, Qi L, Chen R, He J, Liu Z, Wang W, Tu C and Li Z: Circular RNAs in osteoarthritis: indispensable regulators and novel strategies in clinical implications. Arthritis Res Ther 23: 23, 2021

51. Chen C: Serum hsa circ 101178 as a Potential Biomarker for Early Prediction of Osteoarthritis. Clin Lab: Aug 1, 2020 (Epub ahead of print). doi: 10.7754/Clin.Lab.2020.191251.

52. Yao T, Yang Y, Xie Z, Xu Y, Huang Y, Gao J, Shen S, Ye H, Iranmanesh Y, Fan S, et al: Circ0083429 regulates osteoarthritis progression via the Mir-346/SMAD3 axis. Front Cell Dev Biol 8: 579945, 2021.

53. Zhou Z, Ma J, Lu J, Chen A and Zhu L: Circular RNA CircCDH13 contributes to the pathogenesis of osteoarthritis via CircCDH13/miR-296-3p/PTEN axis. J Cell Physiol 236: 3521-3535, 2021.

54. De Bari C and Roelofs AJ: Stem cell-based therapeutic strategies for cartilage defects and osteoarthritis. Curr Opin Pharmacol 40: 74-80, 2018.

55. Pittenger MF, Mackay AM, Beck SC, Jaiswal RK, Douglas R, Mosca JD, Moorman MA, Simonetti DW, Craig S and Marshak DR: Multilineage potential of adult human mesenchymal stem cells. Science 284: 143-147, 1999.

56. Demoor M, Ollitrault D, Gomez-Leduc T, Bouyoucef M, Hervieu M, Fabre H, Lafont J, Denoix JM, Audigié F, Mallein-Gerin F, et al: Cartilage tissue engineering: Molecular control of chondrocyte differentiation for proper cartilage matrix reconstruction. Biochim Biophys Acta 1840: 2414-2440, 2014.

57. Yang L, Bin Z, Hui S, Rong L, You B, Wu P, Han X, Qian H and $\mathrm{Xu} \mathrm{W}$ : The role of CDR1as in proliferation and differentiation of human umbilical cord-derived mesenchymal stem cells. Stem Cells Int 2019: 2316834, 2019.

58. Tu C, He J, Chen R and Li Z: The Emerging role of exosomal non-coding RNAs in musculoskeletal diseases. Curr Pharm Des 25: 4523-4535, 2019.

59. Pegtel DM and Gould SJ: Exosomes. Annu Rev Biochem 88: 487-514, 2019.

60. Staubach S, Bauer FN, Tertel T, Börger V, Stambouli O, Salzig D and Giebel B: Scaled preparation of extracellular vesicles from conditioned media. Adv Drug Deliv Rev 177: 113940, 2021.

61. Zoulikha M, Xiao Q, Boafo GF, Sallam MA, Chen Z and He W: Pulmonary delivery of siRNA against acute lung injury/acute respiratory distress syndrome. Acta Pharm Sin B: Aug 12, 202 (Epub ahead of print). doi: 10.1016/j.apsb.2021.08.009.

62. Chang X, Ma Z, Zhu G, Lu Y and Yang J: New perspective into mesenchymal stem cells: Molecular mechanisms regulating osteosarcoma. J Bone Oncol 29: 100372, 2021. 
63. van der Pol E, Böing AN, Harrison P, Sturk A and Nieuwland R: Classification, functions, and clinical relevance of extracellular vesicles. Pharmacol Rev 64: 676-705, 2012.

64. Yang L, Han B, Zhang Z, Wang S, Bai Y, Zhang Y, Tang Y, Du L, Xu L, Wu F, et al: Extracellular vesicle-mediated delivery of circular RNA SCMH1 promotes functional recovery in rodent and nonhuman primate ischemic stroke models. Circulation 142: $556-574,2020$
65. Liu D, Liang YH, Yang YT, He M, Cai ZJ, Xiao WF and Li YS: Circular RNA in osteoarthritis: An updated insight into the pathophysiology and therapeutics. Am J Transl Res 13: 11-23, 2021.

(c) (i) $(9)$ This work is licensed under a Creative Commons Attribution-NonCommercial-NoDerivatives 4.0 International (CC BY-NC-ND 4.0) License. 